University of Nebraska - Lincoln

DigitalCommons@University of Nebraska - Lincoln

$11-1-1982$

\title{
Effect of anisotropy strength on phase transitions in random anisotropy magnets
}

Michael J. O'Shea

University of Nebraska-Lincoln, mjoshea@phys.ksu.edu

David J. Sellmyer

University of Nebraska-Lincoln, dsellmyer@unl.edu

Follow this and additional works at: https://digitalcommons.unl.edu/physicssellmyer

Part of the Physics Commons

O'Shea, Michael J. and Sellmyer, David J., "Effect of anisotropy strength on phase transitions in random anisotropy magnets" (1982). David Sellmyer Publications. 156.

https://digitalcommons.unl.edu/physicssellmyer/156

This Article is brought to you for free and open access by the Research Papers in Physics and Astronomy at DigitalCommons@University of Nebraska - Lincoln. It has been accepted for inclusion in David Sellmyer Publications by an authorized administrator of DigitalCommons@University of Nebraska - Lincoln. 


\title{
Effect of anisotropy strength on phase transitions in random anisotropy magnets
}

\author{
M. J. O'Shea and D. J. Sellmyer \\ Behlen Laboratory of Physics, University of Nebraska, Lincoln, Nebraska 68588
}

\begin{abstract}
ac susceptibility and magnetization measurements are reported for a number of $\mathrm{Gd}$ and $\mathrm{Tb}$ based glasses. Raman magnetic anisotropy effects are observed in both the $\mathrm{Gd}$ and $\mathrm{Tb}$ glasses. The Gd glasses show a transition to an infinite susceptibility state and in some cases a further transition to an hysteretic state at low temperatures in which field-cooling and magnetic viscosity effects are observed. The Tb glass shows only a small speromagnetic peak at low temperatures. The ratio of anisotropy strength to exchange was varied in the Gd glasses by alloying, and the effect of this on the resulting magnetic states is discussed.
\end{abstract}

PACS numbers: $75.30 . \mathrm{Gw}, 75.30 . \mathrm{Kz}, 75.50 . \mathrm{Kj}$

\section{INTRODUCTION}

Rare-earth-rich metallic glasses show a variety of interesting magnetic properties due to the presence of a random magnetic anisotropy (RMA). The simplest Hamiltonian incorporating this RMA may be written [1]

$$
H=-J \sum_{i j} \vec{J}_{i} \cdot \vec{J}_{j}-D \sum_{i}\left(\hat{n}_{i} \cdot \vec{J}_{j}\right)^{2}
$$

where the first term represents the exchange of strength $J>0$; i.e., ferromagnetic, and the second term represents the RMA of strength $D>0$; i.e., un $i$ axial. $\hat{n}_{j}$ is a unit vector which varies at random from site to site describing the uniaxial anisotropy. Aharony and Pytte [AP] have determined the magnetic equation of state for a system with this Hamiltonian in the limit of small $D / J$ and small $\mathrm{H} / \mathrm{M}$ [3]. In this model the RMA is strong enough to destroy the magnetic order but does not destroy the infinite susceptibility. Recently we have reported [2] on an infinite susceptibility state in $\mathrm{Gd}_{72} \mathrm{Ga}_{18} \mathrm{~B}_{10}$ and the onset of hysteresis at low temperatures.

For Gd, an S-state ion, the single-ion anisotropy strength is expected to be very small. However for $T b$ and other rare-earth ions, $D$ is expected to be quite large so that the theory of AP is not likely to be applicable. In our Gd-rich alloys we find an effective anisotropy constant (K) which is orders of magnitude larger than crystalline Gd. In addition we have found that $K$ can be changed in these glasses by varying the glass formers. In th is paper we report on the effects of changing the anisotropy strength on the phase transitions occurring in $\mathrm{Gd}_{72} \mathrm{Ga}_{18}{ }_{10}$ where $T=B, N i, A l$ and $\mathrm{Gd}_{58} T_{32} B_{10}$ where $T=G a, N i, A l$ and $\mathrm{Tb}_{72}{ }^{\mathrm{Ga}}{ }_{18}{ }^{\mathrm{B}}{ }_{10}$.

\section{EXPERIMENTAL DETAILS}

The amorphous alloys of this work were prepared by the splat-cooling technique. X-ray diffractograms were taken on all of the samples and no evidence of crystalline phases was found. Also the measured $A C$ susceptibility, $X_{A C}\left(=d M / d H_{a} ; H_{a}\right.$ is the applied field) showed no structure above the transition temperature which one observes if there are crystalline Gd-rich phases present. The samples were in the form of strips of dimensions $\sim 4 \mathrm{~mm} \times 1 \mathrm{~mm}$ oriented in order to reduce demagnetizing effects. The AC sus- ceptibility was measured in the temperature range 4.2 - $300 \mathrm{~K}$ at a frequency of $280 \mathrm{~Hz}$ in RMS applied fields of less than 0.01 0e. The high-field magnetization data were measured in fields up to $80 \mathrm{kOe}$ using a superconducting solenoid and vibrating sample magnetometer at temperatures of $4.2 \mathrm{~K}$ and above.

\section{RESULTS}

$X_{A C}$ curves for the series $G_{58}{ }^{T}{ }_{32} B_{10}$ where $T=$ $\mathrm{Ga}, \mathrm{Ni}$, and $\mathrm{AI}$ are shown in Fig. 1 and are limited by the demagnetization factor, $N$, below a certain temperature $T_{C}$. The true susceptibility $\times(=\mathrm{dM} / \mathrm{dH}$; $\left.H=H_{a}-N M\right)$ therefore diverges below $T_{c}$. In addition both the $\mathrm{Ni}$ and $\mathrm{Al}$ glasses show a further transition at some lower temperature $T_{h}$ to an hysteretic phase. $T_{h}$ was calculated from the construction shown in Fig. Ic. An infinite susceptibility phase was also observed in each of the glasses of the series $\mathrm{Gd}_{72} \mathrm{Ga}_{18} \mathrm{~T}_{10}$ where $\mathrm{T}=\mathrm{B}, \mathrm{Ni}, \mathrm{Al}$ and all of these alloys show a transition to an hysteretic phase at low temperatures. In contrast $x_{A C}$ for the glass $\mathrm{Tb}_{72} \mathrm{Ga}_{18} \mathrm{~B}_{10}$ is not limited by the demagnetization factor but rather shows a small peak (Fig. 1 ) indicating a transition to a speromagnetic state. The speromagnetic state is a frozen-in random spin structure much like a spin-glass state; the difference is that the former is produced by the presence of an RMA while the latter is produced by the presence of antiferromagnetic interactions. The temperatures $T_{C}$ and $T_{h}$ for each of these alloy series are summarized in Table 1.

The effective anisotropy constant $(K)$ values for each of these alloys were calculated from their high-field magnetization curves using the magnetization area method. [4] Examples of the magnetization curves for the $\mathrm{Gd}_{58}{ }_{32}{ }^{\mathrm{B}}{ }_{10}$ glasses and the

$\mathrm{Tb}_{72} \mathrm{Ga}_{18} \mathrm{~B}_{10} \mathrm{glass}$ are shown in $\mathrm{Fig}$. 2. Also magnetic isotherms in the form of Arrott plots are shown in $\mathrm{Fig}$. 3 for the $\mathrm{Gd}_{58} \mathrm{Al}_{32} \mathrm{~B}_{10}$ glass. The exchange strength $J_{1}(=2 J$ where $z$ is the coordination number) was calculated using mean field theory from $T_{C}$ for both Gd series and from $T_{h}$ for the Tb glass. Defining $D_{1}=\mathrm{K} / \mathrm{nk}_{\mathrm{B}}$ where $\mathrm{n}$ is the number density of mag- 


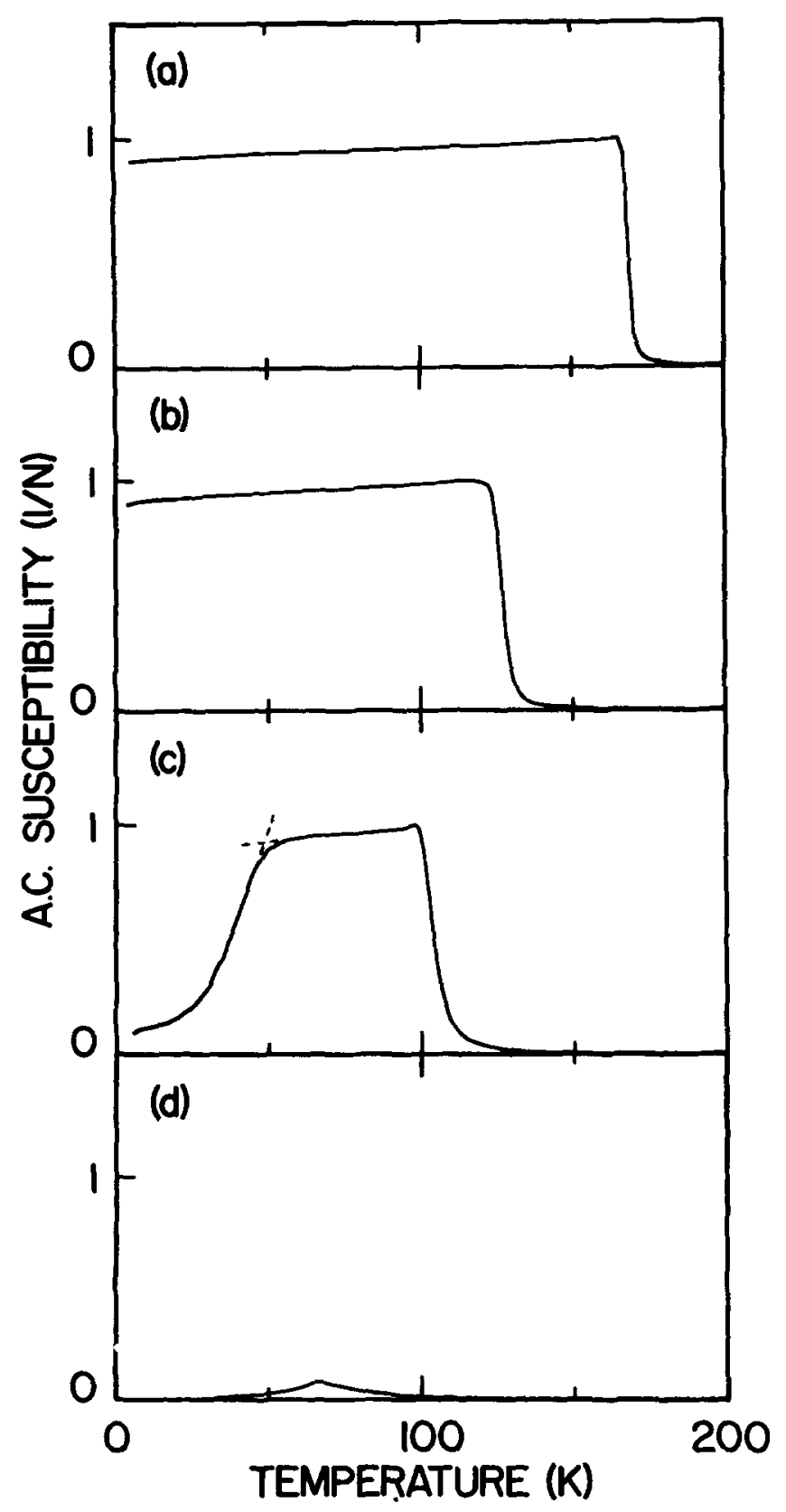

Fig. 1. The $A C$ susceptibility of the series $\mathrm{Gd}_{58}{ }^{\mathrm{T}} 32^{\mathrm{B}} 10$ where $\mathrm{T}$ is (a) $\mathrm{Ga}$, (b) $\mathrm{Ni}$, (c) $\mathrm{Al}$; and also (d) $\mathrm{Tb}_{72} \mathrm{Ga}_{18^{\mathrm{B}} 10^{\circ}}$

netic ions, the resulting $D_{1}$ and $J_{1}$ values are summarized in Table 1.

We have also observed field-cooling and magnetic viscosity effects in the hysteretic state of many of these glasses. These effects will be discussed in a later publication.

\section{DISCUSSION}

The source of RMA in the Tb glass is the random electric field gradients generated by the positional disorder of the Tb ions. The source of this anisotropy in the Gd glasses is somewhat uncertain but a possibility is exchange anisotropy of the type discussed by Fert and Levy [5].
From Table 1 the overall effect of increasing $D_{1} / d_{1}$ in both $\mathrm{Gd}$ series is first to introduce a low temperature hysteretic transition at temperature $T_{h}$. As $D_{1} / J_{1}$ is increased further $T_{h}$ is moved to higher temperatures.

Also, as shown in Fig. 2, as $D_{1} / J_{1}$ increases the magnetization decreases monotonically. For a speromagnetic system which has been perturbed by the field into an asperomagnetic state with a hemisphercal fan, the magnetization would be $g J / 2$ per spin $\left(M / M_{0}=\frac{1}{2}\right)$. Fig. 2 thus suggests that the magnetic

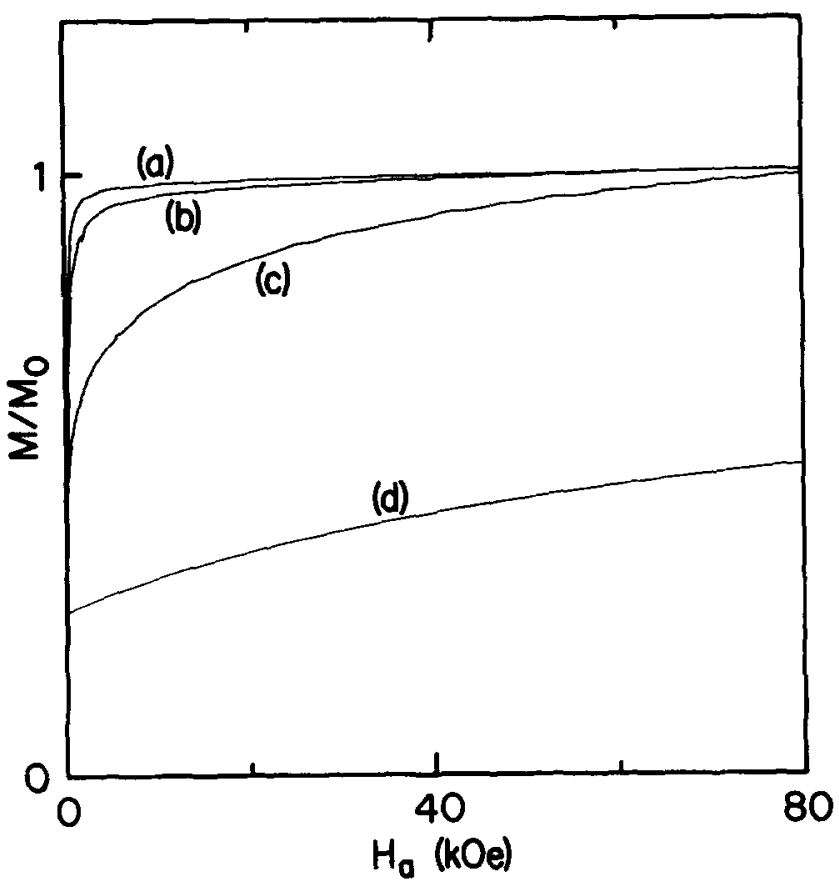

Fig. 2. High-field magnetization curves for the series $\mathrm{Gd}_{58} \mathrm{~T}_{32} \mathrm{~B}_{10}$ where $\mathrm{T}$ is (a) $\mathrm{Ga}$, (b) $\mathrm{Ni}$, (c) $\mathrm{Al}$; and also (d) $\mathrm{Tb}_{72} \mathrm{Ga}_{18} \mathrm{~B}_{10}{ }^{\circ} \mathrm{M}_{0}$ is the saturation magnetization corresponding to the complete alignment of the spins.

TABLE 1

\begin{tabular}{|c|c|c|c|c|c|}
\hline & $T_{c}(K)$ & $T_{h}(k)$ & $D_{1}(K)$ & $J_{1}(K)$ & $\mathrm{D}_{1} / J_{1}$ \\
\hline $\mathrm{Gd}_{58^{G a}}{ }_{32^{B}}{ }_{10}$ & 166 & - & 0.61 & 15.8 & 0.04 \\
\hline $\mathrm{Gd}_{58^{\mathrm{Ni}}}{ }_{32}^{\mathrm{B}} 10$ & 124 & 9 & 0.93 & 8.9 & 0.105 \\
\hline $\left.\mathrm{Gd}_{58^{A}}\right]_{32^{B}}{ }_{10}$ & 98 & 50 & 4.75 & 9.7 & 0.49 \\
\hline $\mathrm{Gd}_{72}{ }^{\mathrm{Ga}} 18^{\mathrm{B}} 10$ & 123 & 41 & 2.90 & 11.7 & 0.25 \\
\hline $\mathrm{Gd}_{72} \mathrm{Ga}_{18^{\mathrm{Ni}}} 10$ & 116 & 26 & 3.22 & 11.0 & 0.29 \\
\hline $\mathrm{Gd}_{72} \mathrm{Ga}_{18^{\mathrm{Al}}}{ }_{10}$ & 127 & 27 & 3.15 & 12.1 & 0.26 \\
\hline${ }^{\mathrm{Tb}}{ }_{72}{ }^{\mathrm{Ga}}{ }_{18^{\mathrm{B}}} 10$ & - & 66 & 11.6 & 2.36 & 4.92 \\
\hline
\end{tabular}




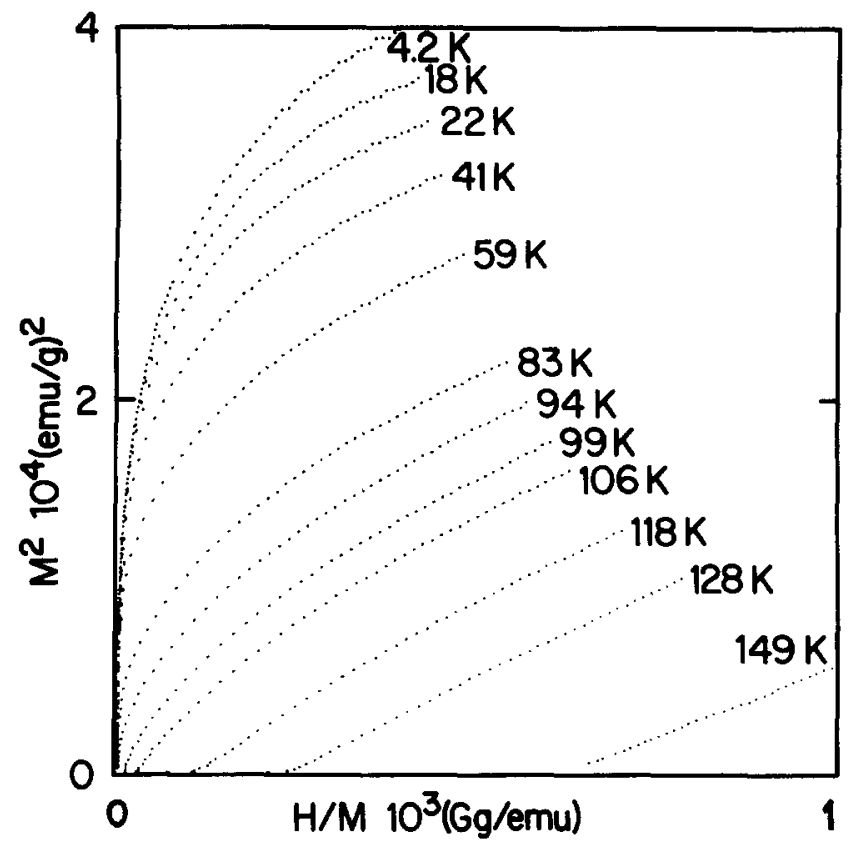

Fig. 3. Magnetic isotherms in the form of Arrott plots for $\mathrm{Gd}_{58} \mathrm{Al}_{32} \mathrm{~B}_{10}$. Here $\mathrm{H}$ is the applied field. Correcting for demagnetizing effects (see text) results in a very small shift of the curves to the left $(0.006 \mathrm{x}$ $\left.10^{3} \mathrm{Gg} / \mathrm{emu}\right)$.

state is increasingly driven towards speromagnetism as $D_{1} / J_{1}$ increases. The increase in $T_{h}$ with $D_{1} / J_{1}$ is evidence that thermal excitations of spins over anisotropy barriers are required to break up the low temperature hysteretic state.

At large $D_{1} / d d_{1}$ values the infinite susceptibility

state is suppressed and there is a direct transition

from the paramagnetic state to the speromagnetic

state as is evidenced by the Tb glass.

With these data we can suggest a qualitative phase diagram as shown in Fig. 4. The dashed line indicates the possibility of a phase boundary between an inf $i$ nite susceptibility phase with no order (IS(AP)) and an infinite susceptibility phase with ferromagnetic domains (IS(FM)). The speromagnetic phase may also become an hysteretic ferromagnet at low $0_{1} / J_{1}$ but we have not indicated this possibility in the phase diagram.

In future work we intend to make alloys whose $\mathrm{D}_{1} / J_{1}$ values approach the tricritical point in the phase diagram and also to explore the possibility of a phase boundary in the infinite susceptibility part of the phase diagram. We are pursuing a critical exponent analysis of several of these alloys at present.

We would like to thank Professor Z. D. Chen for assistance in taking the AC susceptibility data, J. Nabity for making our samples and Dr. S. G. Cornelison for many useful discussions. We are grateful to the National Science Foundation for support of this work under Grant number DMR-8110520.

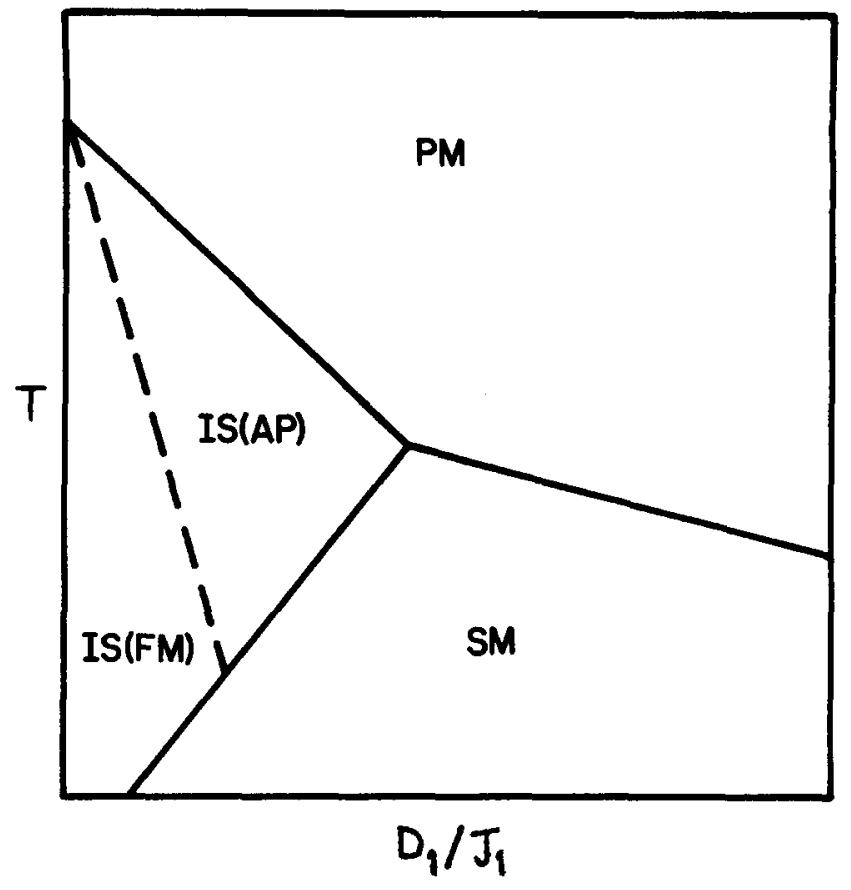

Fig. 4. Schematic phase diagram for a RMA system. The abbreviations are PM - paramagnetic; SM - speromagnetic; IS (AP) - Aharony and Pytte infinite susceptibility state; IS(FM) - infinite susceptibility ferromagnetic state with a domain structure. REFERENCES

(1) R. Harris, M. Plischke and M. J. Zuckerman, Phys. Rev. Lett. 31, 160 (1973).

(2) M. J. O'Shea and D. J. Selimyer, Bull. Am. Phys. Soc. 27, 412 (1982), and to be published.

(3) A. Aharony and E. Pytte, Phys. Rev. Lett. 45 $1583(1980)$.

(4) See for example G. Hadjipanayis, D. J. Sellmyer and B. Brandt, Phys. Rev. B 23, 3349 (1981).

(5) A. Fert and P. M. Levy, Phys. Rev. Lett. 44, $1538(1980)$. 